\title{
Using Fuzzy Sets for Coarseness Representation in Texture Images
}

\author{
J. Chamorro-Martínez ${ }^{1}$, E. Galán-Perales ${ }^{1}$, J.M. Soto-Hidalgo ${ }^{1}$, and B. \\ Prados-Suárez ${ }^{2 \star}$ \\ 1 Department of Computer Science and Artificial Intelligence, University of Granada, \\ C/ Periodista Daniel Saucedo Aranda s/n, 18071 Granada, Spain \\ \{jesus, elena, soto\}@decsai.ugr.es \\ 2 Department of Computer Science, University of Jaen, C/ Alfonso X El Sabio 28, \\ 23700, Linares, Jaen, Spain \\ belenps@ujaen.es
}

\begin{abstract}
Texture is a visual feature frequently used in image analysis that has associated certain vagueness. However, the majority of the approaches found in the literature do not either consider such vagueness or they do not take into account human perception to model the related uncertainty. In this paper we model the concept of "coarseness", one of the most important textural features, by means of fuzzy sets and considering the way humans perceive this kind of texture. Specifically, we relate representative measures of coarseness with its presence degree. To obtain these "presence degrees", we collect assessments from polls filled by human subjects, performing an aggregation of such assessments. Thus, the membership function corresponding to the fuzzy set "coarseness" is modelled by using as reference set the representative measures and the aggregated data.
\end{abstract}

Keywords: Image features, texture features, fuzzy texture, visual coarseness.

\section{Introduction}

Texture is being increasingly recognized as an important cue for the analysis of natural imagery. It is one of the most difficult visual features to be characterized due to the imprecission of the concept itself. In fact, there is not an accurate definition for the concept of texture but some widespread intuitive ideas. In this way, texture is described by some authors as local changes in the intensity patterns or gray tones. Other authors consider texture as a set of basic items called texels (or texture primitives), arranged in a certain way [1]. Moreover, it is usual for humans to describe visual textures according to some "textural concepts" like coarseness, orientation, regularity [2]. To describe such concepts, linguistic labels are used (e.g. coarse or fine can be used to describe coarseness).

\footnotetext{
* This work has been partially supported by "Consejera de Innovacin Ciencia y Empresa" under TIC-1570 project
} 

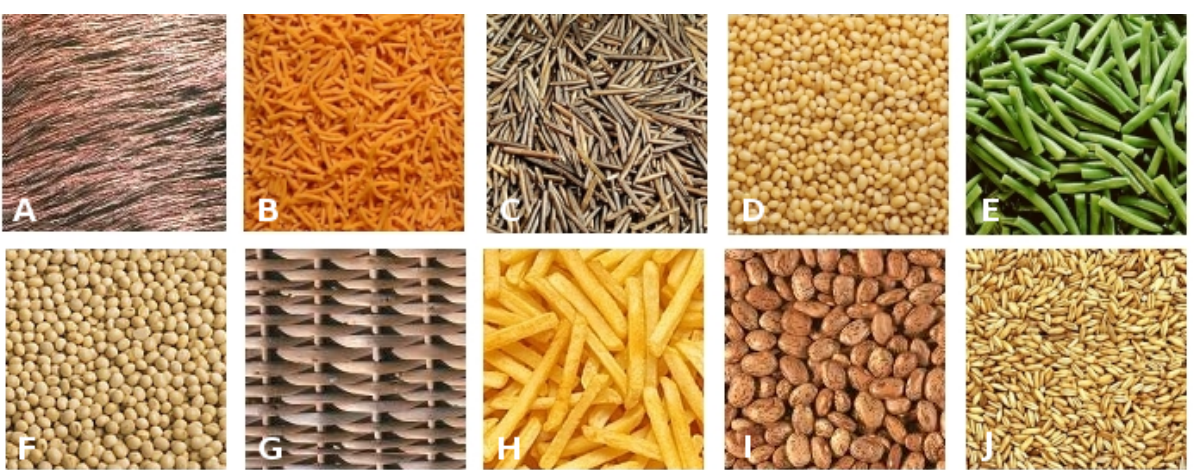

Fig. 1. Some examples of images with different degrees of fineness

The own imprecision of the concept of texture suggests to use representation models that incorporate the uncertainty. Nevertheless, the majority of the approaches that can be found are crisp proposals [3] where uncertainty is not properly taken into account. To deal with the imprecission relative to visual texture, there are some approaches which introduce the use of fuzzy logic. However, in many of these approaches, fuzzy logic is usually applied just during the process but the output do not habitually model the imprecission (being often a crisp one). Examples of this fact are frequently found in the literature, like those approaches that use texture to perform image segmentation. A lot of these approaches use fuzzy clustering [4], fuzzy rules [5], etc. but most of them have in common that the obtained result is crisp, no matter the intermediate representation for uncertainty used.

In this paper we focus our study on coarseness, one of the textural properties most used in the literature which allows to distinguish between fine and coarse textures. In fact, the concept of texture is usually associated to the presence of fineness. A fine texture can be considered as small texture primitives with big gray tone differences between neighbour primitives (e.g. the image in figure $1(\mathrm{~A})$ ). On the contrary, if texture primitives are bigger and formed by several pixels, it is a coarse texture (e.g. the image in figure 1(I)).

In our approach, we propose to model fineness by means of fuzzy sets to deal with the problem of imprecision found in texture characterization. To do this, two questions will be faced: what reference set should be used for the fuzzy set, and how to obtain the related membership functions. To solve the first question, a set of measures will be automatically computed from the texture image. To answer the second question, functional relationship between a certain measure and the presence degree of a textural concept related to it will be found considering human perception.

The rest of the paper is organized as follows. In section 2 we introduce our methodology to obtain the fuzzy sets related to fineness textural concept. In section 3 we show the results of applying the model and the main conclusions and future work are sumarized in section 4 . 


\section{$2 \quad$ Fineness Modelling}

There are many measures over the literature that, given an image, capture the fineness (or coarseness) presence in the sense that the greater the value given by the measure, the greater the perception of texture. However, there is no perceptual relationship between the value given by these measures and the degree in which the humans perceive the texture. Thus, given a certain value calculated by applying a measure to an image, there is not an immediate way to decide whether there is a fine texture, a coarse texture or something intermediate (i.e. there is not a textural interpretation).

To face this problem, we propose to model the fineness perception as a fuzzy set defined on the domain of a given measure. Let $\mathcal{P}=\left\{P_{1}, \ldots, P_{K}\right\}$ be a set of measures of fineness (e.g. $\mathcal{P}=\{$ EdgeDensity, Variance, Entropy $\}$ ) and let $\mathcal{T}_{k}$ be a fuzzy set defined on the domain of $P_{k} \in \mathcal{P}$ representing the concept of "fineness". Thus, the membership function associated to $\mathcal{T}_{k}$ will be defined as ${ }^{1}$

$$
\mathcal{T}_{k}: \mathbb{R} \rightarrow[0,1]
$$

where a value of 1 will mean fineness presence while a value of 0 will mean no fineness presence (i.e. coarseness presence).

In this section, given a measure $P_{k} \in \mathcal{P}$, we propose to obtain $\mathcal{T}_{k}$ by finding a functional relationship between $P_{k}$ and the perception degree of fineness. To do it, we will use a set $\mathcal{I}=\left\{I_{1}, \ldots, I_{N}\right\}$ of $N$ images that fully represent the different degrees of fineness. Thus, for each image $I_{i} \in \mathcal{I}$, we will obtain (a) a human assessment of the fineness degree perceived, noted as $v^{i}$, which will be collected by means of a poll with human subjects (section 2.1), and (b) a value calculated applying the measure $P_{k} \in \mathcal{P}$ to the image $I_{i}$, noted as $m_{k}^{i}$ (section 2.2). From the multiset $\left\{\left(m_{k}^{1}, v^{1}\right), \ldots,\left(m_{k}^{N}, v^{N}\right)\right\}$, the membership function $\mathcal{T}_{k}$ will be estimated (section 2.3).

\section{$2.1 \quad$ Assessment collection}

In this section, the way to obtain a vector $\Gamma=\left[v 1, \ldots, v^{N}\right]$ of the assessments of the perception degree of fineness from the image set $\mathcal{I}=\left\{I_{1}, \ldots, I_{N}\right\}$ will be described. Thus, firstly the image set $\mathcal{I}$ will be selected. After that, a poll which allows to get assessments of the perception degree of fineness will be designed. These assessments will be obtained for each image in $\mathcal{I}$, so an aggregation of the different assessments will be performed.

The texture image set A set $\mathcal{I}=\left\{I_{1}, \ldots, I_{N}\right\}$ of $N=80$ images representative of the concept of fineness has been selected. Figure 1 shows some images extracted from the set $\mathcal{I}$. The selection was done to cover the different perception degrees of fineness with a representative number of images. Furthermore,

\footnotetext{
${ }^{1}$ To simplify the notation, as it is usual in the scope of fuzzy sets, we will use the same notation $\mathcal{T}_{k}$ for the fuzzy set and for the membership function that defines it
} 
the images have been chosen so that as far as possible, just one perception degree of fineness is perceived.

The poll Given the image set $\mathcal{I}$, the next step is to obtain assessments about the perception of fineness from a set of subjects. From now on we shall note as $\Theta^{i}=\left[o_{1}^{i}, \ldots, o_{L}^{i}\right]$ the vector of assessments obtained from $L$ subjects for the image $I_{i}$. To get $\Theta^{i}$, subjects will be asked to assign images to classes, so that each class has associated a perception degree of fineness.

In particular, 20 subjects have participated in the poll and 9 classes have been considered. The first nine images in figure 1 show the nine representative images for each class used in this poll. It should be noticed that the images are decreasingly ordered according to the presence degree of the fineness concept. The first class (Figure 1(A)) represents a presence degree of 1 while the ninth class (Figure 1(I)), represents a presence degree of 0 . The rest of the classes (Figure $1(\mathrm{~B})-(\mathrm{H}))$ represent degrees in the interval $(0,1)$.

As result, a vector of 20 assessments $\Theta^{i}=\left[o_{1}^{i}, \ldots, o_{20}^{i}\right]$ is obtained for each image $I_{i} \in \mathcal{I}$. The degree $o_{j}^{i}$ associated to the assessment given by the subject $S_{j}$ to the image $I_{i}$ is computed as $o_{j}^{i}=(9-k) * 0.125$, where $k \in\{1, \ldots, 9\}$ is the index of the class $C_{k}$ to which the image is assigned by the subject.

Assessment aggregation Our aim at this point is to obtain, for each image in the set $\mathcal{I}$, one assessment $v^{i}$ that summarizes the assessments $\Theta^{i}$ given by the different subjects about the presence degree of fineness.

To aggregate opinions we have used an OWA operator guided by a quantifier [7]. Concretely, the quantifier "the most" has been employed, which allows to represent the opinion of the majority of the polled subjects. This quantifier is defined as

$$
Q(r)=\left\{\begin{array}{lr}
0 & \text { if } r<a \\
\frac{r-a}{b-a} \text { if } a \leq r \leq b \\
1 & \text { if } r>b
\end{array}\right.
$$

with $r \in[0,1], a=0.3$ and $b=0.8$. Once the quantifier $Q$ has been chosen, the weighting vector of the OWA operator can be obtained following Yager [7] as $w_{j}=Q(j / L)-Q((j-1) / L), j=1,2, \ldots, L$. According to this, for each image $I_{i} \in \mathcal{I}$, the vector $\Theta^{i}$ obtained from $L$ subjects will be aggregated into one assessment $v^{i}$ as follows:

$$
v^{i}=w_{1} \hat{o}_{1}^{i}+w_{2} \hat{o}_{2}^{i}+\ldots+w_{L} \hat{o}_{L}^{i}
$$

where $\left[\hat{o}_{1}^{i}, \ldots, \hat{o}_{L}^{i}\right]$ is a vector obtained by ranking in nonincreasing order the values of the vector $\Theta^{i}$.

\subsection{Fineness measures}

In our proposal, the fuzzy set $\mathcal{T}_{k}$ will be defined on the domain of a certain measure of fineness $P_{k}$. Due to the fact that there are many measures in the 
literature that characterize the presence of fine texture, a selection of a suitable set $\mathcal{P}=\left\{P_{1}, \ldots, P_{K}\right\}$ is needed. In this paper, we propose to use the 18 measures shown in the first column of table 1 (that includes classical statistical measures well known in the literature, measures in the frequency domain, etc.).

From the measures shown in table 1 , some will have better ability to represent fineness while others will be worse. Thus, the question of what ability a measure has to discriminate different presence degrees of fineness needs to be solved, i.e. how many classes can $P_{k}$ actually discriminate. To face this question, we propose to analyze each $P_{k} \in \mathcal{P}$ by applying a set of multiple comparison tests following the algorithm 1 . This algorithm starts with an initial partition and iteratively joins clusters until a partition in which all classes are distinguishable is achieved. In our proposal, the initial partition will be formed by the 9 classes used in our poll (where each class will contain the images assigned to it by the majority of the subjects), as $\delta$ the Euclidean distance between the centroids of the involved classes will be used, as $\phi$ a set of 5 multiple comparison tests will be considered (concretely, the tests of Scheffé, Bonferroni, Duncan, Tukey's least significant difference, and Tukey's honestly significant difference [8]), and finally the number of positive tests to accept distinguishability will be fixed to $N T=3$.

From now on, we shall note as $C_{1}^{k}, C_{2}^{k}, \ldots, C_{N C_{k}}^{k}$ the $N C_{k}$ classes that can be discriminated by $P_{k}$. For each $C_{r}^{k}$, we will note as $\bar{c}_{r}^{k}$ the class representer value and as $\bar{v}_{r}^{k}$ the presence degree of fineness associated to $C_{r}^{k}$. In this paper, we propose to compute $\bar{c}_{r}^{k}$ as the mean of the measure values in the class $C_{r}^{k}$ and $\bar{v}_{r}^{k}$ as the mean of the presence degrees of fineness associated to the classes grouped into $C_{r}^{k}$.

In the case of fineness, table 1 shows the results obtained by applying the proposed algorithm 1 with the different measures considered in this paper. The second column of this table shows the number of classes that each measure can discriminate and the third column shows how the initial classes have been grouped. Moreover, the columns from fourth to seventh show the representer values $\bar{c}_{r}^{k}$ and $\bar{v}_{r}^{k}$ associated to each cluster. It can be noticed that the measure that can discriminate a higher number of classes is $\mathrm{ED}$, while the majority of the measures discern 2 or 3 classes. Furthermore, the measures in the last 4 rows of table 1 cannot discriminate more than one class, being discarded for later analysis because of their little representativeness of fineness.

In reference to the the classes joint, note that for most cases the initial classes 1 and 9 have not been grouped into a greater cluster except in the case of the measures FD and Tamura. For these measures, the classes 1, 2 and 3 were grouped into one cluster, which means they cannot discriminate properly the presence degree of fineness when the texture is getting fine.

\subsection{Obtaining the membership function}

In this section we will deal with the problem of obtaining the membership function for the fuzzy set $\mathcal{T}_{k}$. Concretely, we propose to define such membership 


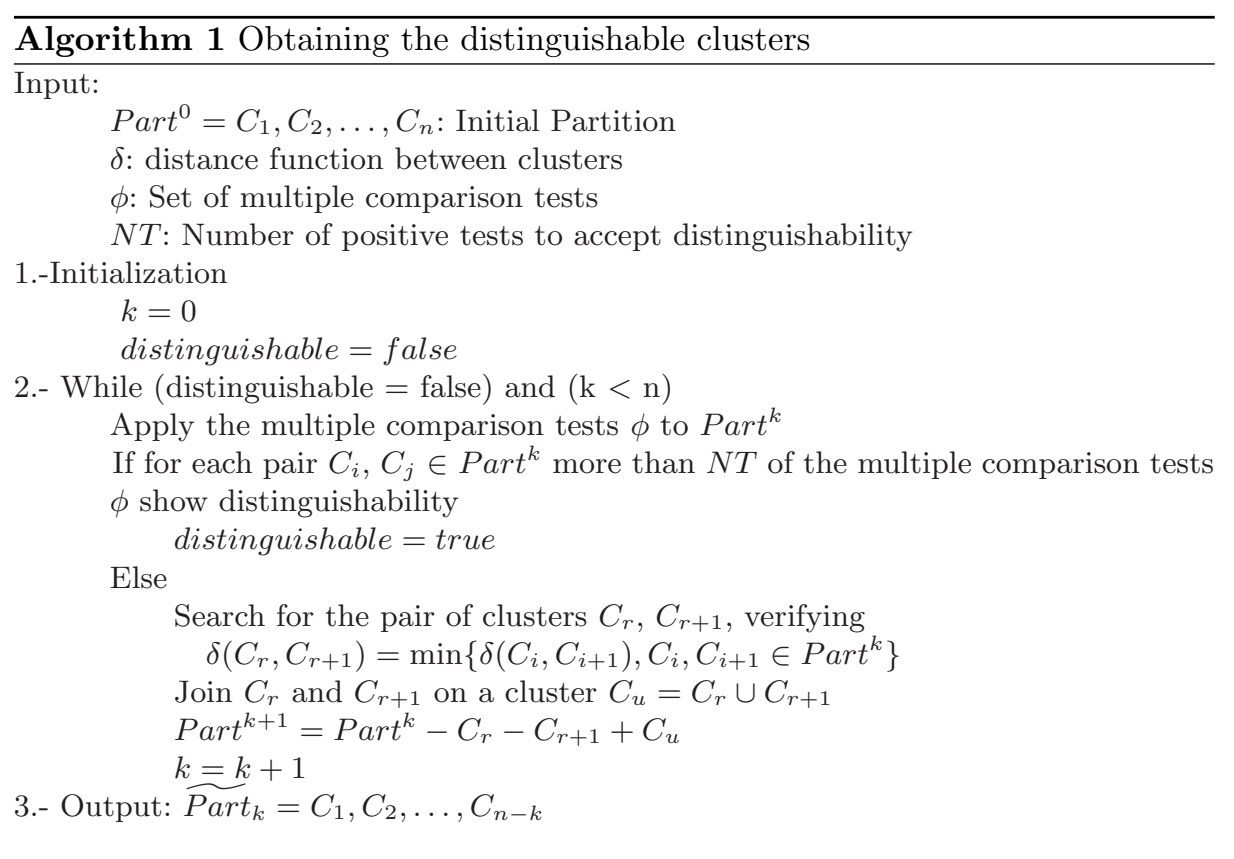

function as a linear spline that associates the values given by a certain measure with the assessments given by the human subjects, i.e.:

$$
\mathcal{T}_{k}(x)=\left\{\begin{array}{lr}
0 & x \leq x_{1} \\
f^{1}(x) & x \in\left(x_{1}, x_{2}\right] \\
f^{2}(x) & x \in\left(x_{2}, x_{3}\right] \\
\vdots & \vdots \\
1 & x>x_{N C_{k}}
\end{array}\right.
$$

with $f^{r}(x)$ being a straight line defined as $f^{r}(x)=a_{1}^{r} x+a_{0}^{r}$.

To obtain the parameters of equation 4 , the classes resulting from the application of the algorithm 1 (that in fact, were obtained considering the ability of the measure to distinguish the classes originally given by the subjects) will be used. In this paper, the spline knots will be chosen considering the class representers, i.e., $x_{r}=\bar{c}_{r}^{k}$ with $x_{r}$ being the centroid of $C_{r}^{k}$. The function $f^{r}(x)$ will be obtained as the straight line defined between the points $\left(\bar{c}_{r}^{k}, \bar{v}_{r}^{k}\right)$ and $\left(\bar{c}_{r+1}^{k}, \bar{v}_{r+1}^{k}\right)$, with $\bar{v}_{r}^{k}$ being the fineness degree of presence related to the cluster $C_{r}^{k}$. Thus, the parameters $a_{1}^{r}$ and $a_{0}^{r}$ of $f^{r}(x)$ are computed as $a_{1}^{r}=\frac{\bar{v}_{r+1}^{k}-\bar{v}_{r}^{k}}{\bar{c}_{r+1}^{k}-\bar{c}_{r}^{k}}$ and $a_{0}^{r}=\bar{v}_{r}^{k}-\bar{c}_{r}^{k} a_{1}^{r}$, respectively.

The way the spline is defined allows to ensure that, for the representer values $\bar{c}_{r}^{k}$ of each class, the membership function returns the mean assessment given by the subjects to that class (i.e., $\left.\mathcal{T}_{k}\left(\bar{c}_{r}^{k}\right)=\bar{v}_{r}^{k}\right)$. From this point of view, $f^{r}(x)$ may 


\begin{tabular}{|l|c|c|c|c|c|c|c|}
\hline Measures & $N C_{k}$ & Classes & $\left(\overline{c_{4}}, \overline{v_{4}}\right)$ & $\left(\overline{c_{3}}, \overline{v_{3}}\right)$ & $\left(\overline{c_{2}}, \overline{v_{2}}\right)$ & $\left(\overline{c_{1}}, \overline{v_{1}}\right)$ & $\mathrm{RMSE}$ \\
\hline Tamura[9] & 3 & $\{1-3,4-8,9\}$ & - & $(2.76,1)$ & $(3.28,0.375)$ & $(3.61,0)$ & 0.239 \\
FD[10] & 3 & $\{1-3,4-8,9\}$ & - & $(3.04,1)$ & $(2.65,0.38)$ & $(2.39,0)$ & 0.239 \\
ED[11] & 4 & $\{1,2-4,5-8,9\}$ & $(0.374,1)$ & $(0.34,0.75)$ & $(0.31,0.31)$ & $(0.269,0)$ & 0.280 \\
Correlation[12] & 3 & $\{1,2-8,9\}$ & - & $(0.154,1)$ & $(0.579,0.5)$ & $(0.817,0)$ & 0.283 \\
Weszka[13] & 3 & $\{1,2-8,9\}$ & - & $(0.153,1)$ & $(0.102,0.5)$ & $(0.051,0)$ & 0.364 \\
Wu[14] & 3 & $\{1,2-8,9\}$ & - & $(31.5,1)$ & $(21.2,0.5)$ & $(10.5,0)$ & 0.366 \\
Amadasun[15] & 2 & $\{1-8,9\}$ & - & - & $(11.1,1)$ & $(30.8,0)$ & 0.469 \\
LH[12] & 2 & $\{1-8,9\}$ & - & - & $(0.102,1)$ & $(0.202,0)$ & 0.481 \\
DGD[16] & 2 & $\{1,2-9\}$ & - & - & $(14.5,1)$ & $(4.1,0)$ & 0.487 \\
SRE[17] & 2 & $\{1-8,9\}$ & - & - & $(0.986,1)$ & $(0.933,0)$ & 0.487 \\
SNE[18] & 2 & $\{1-8,9\}$ & - & - & $(0.785,1)$ & $(0.564,0)$ & 0.493 \\
Newsam[19] & 2 & $\{1-8,9\}$ & - & - & $(19.1,1)$ & $(17.4,0)$ & 0.512 \\
Entropy[12] & 2 & $\{1-8,9\}$ & - & - & $(9.02,1)$ & $(8.42,0)$ & 0.532 \\
Uniformity[12] & 2 & $\{1-8,9\}$ & - & - & $(0.0002,1)$ & $(0.0004,0)$ & 0.566 \\
Contrast[12] & 1 & - & - & - & - & - & - \\
Variance[12] & 1 & - & - & - & - & - & - \\
FMPS[20] & 1 & - & - & - & - & - & - \\
Abbadeni[2] & 1 & - & - & - & - & - & - \\
\hline
\end{tabular}

Table 1. Number of classes, grouped clusters, representers of the obtained classes and RMSE found by applying the membership function related to each measure

be considered as a function that represents the transition between the classes $C_{r}^{k}$ and $C_{r+1}^{k}$.

The above approach has been used to define the membership functions $\mathcal{T}_{k}$, with $k=1, \ldots, 18$ corresponding to the 18 measures considered in this paper. To do it, the class representer values $\bar{c}_{r}^{k}$ and the corresponding assessment values $\bar{v}_{r}^{k}$ shown in table 1 have been used. These functions have been applied to each image $I_{i} \in \mathcal{I}$ and the obtained value has been compared with the one assessed by human subjects. Table 1 shows the RMSE obtained for the different fuzzy sets considered in this paper. This table has been sorted according to least RMSE, where the less the RMSE, the better the performance of the fuzzy set.

\section{Results}

The function $\mathcal{T}_{k}$ obtained for each measure (defined by the parameter values shown in table 1) has been applied to different real images. Table 2 shows three real images with different perception degree of fineness. For each image and each measure, this table shows the value obtained by the related function and the error obtained when comparing this value with the assessment value given by subjects (by computing the difference between both of them). It can be noticed by looking the results shown in this table that our model allows to represent appropriately the perception of fineness. Note that the values given by the different functions are similar to the corresponding assessment degree for most of the obtained functions. This is particularly noticed for extreme fineness degrees of presence 


\begin{tabular}{|c|c|c|c|c|c|c|}
\hline & \multicolumn{2}{|c|}{$\begin{array}{c}\text { Assessment } \\
\text { Value }=0\end{array}$} & \multicolumn{2}{|c|}{$\begin{array}{c}\text { Assessment } \\
\text { Value }=0.5\end{array}$} & \multicolumn{2}{|c|}{$\begin{array}{c}\text { Assessment } \\
\text { Value }=1\end{array}$} \\
\hline Measure & Value & Error & Value & Error & Value & Error \\
\hline Tamura & 0 & 0 & 0.510 & 0.010 & 1 & 0 \\
\hline $\mathrm{FD}$ & 0 & 0 & 0.641 & 0.141 & 1 & 0 \\
\hline $\mathrm{ED}$ & 0 & 0 & 0.406 & 0.094 & 1 & 0 \\
\hline Correlation & 0 & 0 & 0.519 & 0.019 & 1 & 0 \\
\hline Weszka & 0 & 0 & 0.562 & 0.062 & 0.959 & 0.041 \\
\hline $\mathrm{Wu}$ & 0 & 0 & 0.620 & 0.120 & 1 & 0 \\
\hline Amadasun & 0 & 0 & 0.976 & 0.476 & 1 & 0 \\
\hline LH & 0 & 0 & 1.000 & 0.500 & 1 & 0 \\
\hline DGD & 0 & 0 & 0 & 0.500 & 0.952 & 0.048 \\
\hline SRE & 0 & 0 & 1.000 & 0.500 & 1 & 0 \\
\hline SNE & 0 & 0 & 0.929 & 0.429 & 1 & 0 \\
\hline Newsam & 0 & 0 & 0.983 & 0.483 & 1 & 0 \\
\hline Entropy & 0.030 & 0.030 & 1.000 & 0.500 & 1 & 0 \\
\hline Uniformity & 0.518 & 0.518 & 1.000 & 0.500 & 1 & 0 \\
\hline
\end{tabular}

Table 2. Estimated and error values obtained by applying the proposed model to three real images

(first and last columns in table 2) while for intermediate degrees (central column in table 2) higher errors are obtained, specially for those measures found in the last rows (corresponding to measures with high RMSE in table 1).

Table 3 shows a comparative between our model and the assessments obtained from subjects for a mosaic image. The first column of this table shows the mosaic image made by several images, each one with a different increasing perception degree of fineness. The second column shows the number of the subimage considered from left to right and from top to bottom. The third column shows the assessments given by humans for the different images. The fourth column shows the perception degree of fineness obtained by applying our model using the Tamura measure (the one with least RMSE according to table 1). The fifth column shows the difference between the computed degree and the human assessment. In the case of the sixth column we calculate the differences between the assessment given by each subject and the computed degree, and we obtain as error measure the mean from these 20 differences. Finally, the average errors shown in the last row with values of 0.011 and 0.110 show the goodness of our approach to represent the subjectivity found in fineness perception. It can be noticed that our model captures the evolution of the perception degrees of fineness. Let us remark that when very fine textures are found, the assigned value is 


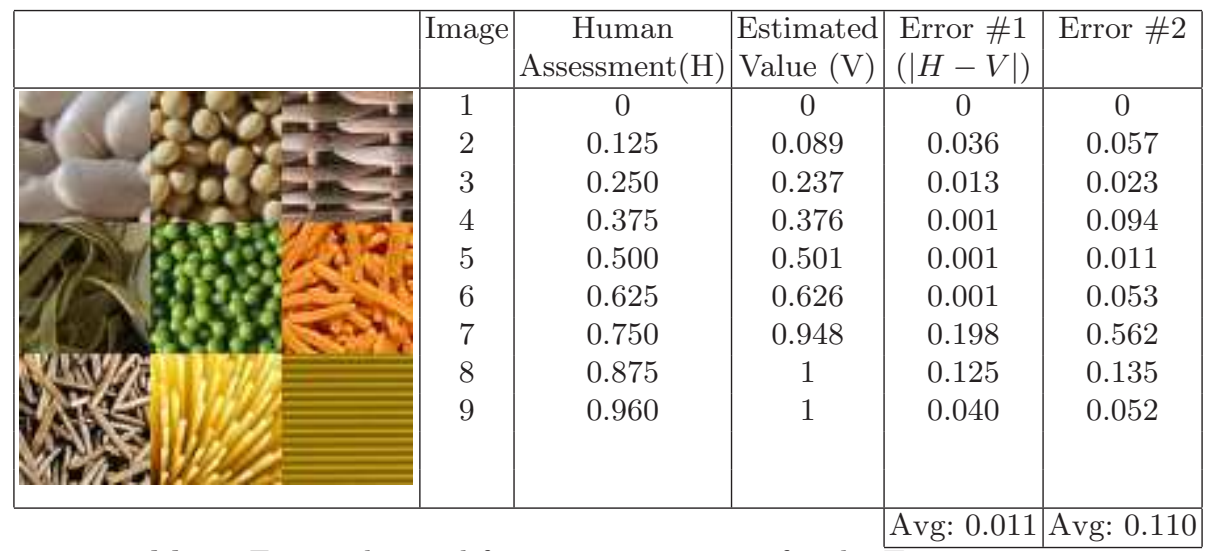

Table 3. Errors obtained from a mosaic image for the Tamura measure

1 which is due to the fact that the Tamura measure had grouped the first classes into one cluster.

\section{Conclusions and future works}

In this paper, a methodology to represent the fineness concept by means of fuzzy sets has been proposed. To define the membership function associated to the fuzzy set, the functional relationship between a given measure (automatically computed over the image) and the perception degree of fineness has been achieved. In order to obtain the perception degree of fineness, a group of human subjects have been polled and their assessments have been aggregated by means of OWA operators. The functions presented in this paper allow to decide whether there is fine, coarse or intermediate texture, splitting the domain into some perceptual meaningful intervals. The results given by our approach show a high level of connection with the assessments given by subjects.

As future work, we will extend the proposed approach to obtain membership functions on $\mathbb{R}^{n}$, i.e. functions on vectors of measures. Furthermore, the performance of the fineness functions will be analyzed in applications like textural classification or segmentation.

\section{References}

1. Shapiro, L.G., Stockman, G.: Image Segmentation. In: Computer Vision. (2001) 297-301

2. Abbadeni, N., Ziou, N., Wang, D.: Autocovariance-based perceptual textural features corresponding to human visual perception. In: Proc. of 15 th International Conference on Pattern Recognition. Volume 3. (2000) 901-904

3. Reed, T.R., Buf, J.H.D.: A review of recent texture segmentation and feature extraction techniques. CVGIP: Image Understanding 57(3) (1993) 359-372 
4. Wang, C., Wang, H., Mei, Q.: Texture segmentation based on an adaptively fuzzy clustering neural network. In: Proc. of 2004 International Conference on Machine Learning and Cybernetics. Volume 2. (2004) 1173-1176

5. Potrebić, M.: Iterative fuzzy rule base technique for image segmentation. In: Proc. of 7th seminar on Neural Network Applications in Elecrical Engineering. (2004) $221-224$

6. Shackelford, A.: A hierachical fuzzy classification approach for high-resolution multispectral data over urban areas. IEEE Transactions on Geoscience and Remote Sensing 41(9) (2003) 1920-1932

7. Yager, R.: On ordered weighted averaging aggregation operators in multicriteria decisionmaking. IEEE Transactions on Systems, Man and Cybernetics 18(1) (1988) 183-190

8. Hochberg, Y., Tamhane, A.: Multiple Comparison Procedures. Wiley (1987)

9. Tamura, H., Mori, S., Yamawaki, T.: Textural features corresponding to visual perception. IEEE Transactions on Systems, Man and Cybernetics 8 (1978) 460473

10. Peleg, S., Naor, J., Hartley, R., Avnir, D.: Multiple resolution texture analysis and classification. IEEE Transactions on Pattern Analysis and Machine Intelligence (4) (1984) 518-523

11. Canny, J.: A computational approach to edge detection. IEEE Transactions on Pattern Analysis and Machine Intelligence 8(6) (1986) 679-698

12. Haralick, R.: Statistical and structural approaches to texture. Proceedings IEEE 67(5) (1979) 786-804

13. Weszka, J., Dyer, C., Rosenfeld, A.: A comparative study of texture measures for terrain classification. IEEE Transactions on Systems, Man and Cybernetics 6 (1976) 269-285

14. Wu, C., Chen, Y.: Statistical feature matrix for texture analysis. CVGIP: Graphical Models and Image Processing 54(5) (1992) 407-419

15. Amadasun, M., King, R.: Textural features corresponding to textural properties. IEEE Transactions on Systems, Man and Cybernetics 19(5) (1989) 1264-1274

16. Kim, S., Choi, K., Lee, D.: Texture classification using run difference matrix. In: Proc. of IEEE 1991 Ultrasonics Symposium. Volume 2. (1991) 1097-1100

17. Galloway, M.: Texture analysis using gray level run lengths. Computer Graphics and Image Processing 4 (1975) 172-179

18. Sun, C., Wee, W.: Neighboring gray level dependence matrix for texture classification. Computer Vision, Graphics and Image Processing 23 (1983) 341-352

19. Newsam, S., Kammath, C.: Retrieval using texture features in high resolution multi-spectral satellite imagery. In: Data Mining and Knowledge Discovery: Theory, Tools, and Technology VI, SPIE Defense and Security. (2004)

20. Yoshida, H., Casalino, D., Keserci, B., Coskun, A., Ozturk, O., Savranlar, A.: Wavelet-packet-based texture analysis for differentiation between benign and malignant liver tumours in ultrasound images. Physics in Medicine and Biology 48 (2003) 3735-3753 